\title{
The Impact of Dirac on 20th Century Science
}

\author{
A. Zichichi \\ CERN, Geneva
}

\begin{abstract}
Preserving a blackboard with its writing is one of many warmhearted gestures that have been made by the Florida State University, Tallahassee in rememberance of Paul Dirac. He supervised students and lectured at the University after his retirement from Cambridge in 1971 until his death at 82 in 1984.

The blackboard is kept in a special collection room in the University's new Paul A.M. Dirac Science Center Library that was dedicated to Dirac last December. The room, which is open to the public, houses a catalogued collection of materials: some were transfered from Cambridge and others were donated by his wife who still lives in

Tallahassee.
\end{abstract}

A panel discussion held after the dedication ceremony allowed several prominent scientists to review Dirac's stupendous achievements. Among them was Professor Zichichi who compared Dirac's contribution to our understanding of the world around us with that of Galilei. He also related a famous story on why the proton was considered for some time as the antiparticle of the electron.

The statue of Paul Dirac, unveiled in December 1989, that is placed in front of the Florida State University library commemorating his name.

\section{The useless particles: from Galilei to Dirac}

Albert Einstein provided us with the first conclusive synthesis of the enormous variety of the world, as it appears to us now. Thanks to him we now know that space and time are not separate entities but manifestations of an unique, four-dimensional quantity. Moreover, energy and mass are equivalent. Finally, in order to understand the structure of the universe where massenergy plays a fundamental rôle, we do not need to worry. Mass-energy is not an additional entity as it is intrinsically contained in the structure and properties of space-time, i.e. its curvature.

With Einstein, the human intellect closed a fascinating intellectual adventure, started by Galileo Galilei who wanted to understand the world around us. He completed Galilei's adventure with his "superb synthesis" of the basic quantities space, time, energy and mass.

It appeared as if nothing else needed to be understood until Dirac's equation opened a new horizon towards a new world - a world which does not exist around us. On this planet where we live, there are no mines of antimatter. In the deepest corner of the ocean or on the highest summit of Everest, we shall never find a piece of antimatter. However, the existence of antimatter is as real as the existence of a stone.

The reason Paul Dirac will rank above Albert Einstein in the history of science is the radical novelty of his thinking. The meaning of the Dirac equation and of its consequences is profound, far-reaching and fascinating, much more so than Einstein's "superb synthesis".

Dirac's prediction of the existence of the antielectron seemed to many scientists to be merely science fiction. Why should we care for particles which do not exist as constituents of the matter around us? What can we expect to find from such speculations, appearing as they do to be philosophical and not directly related with the physical world around us?

Where is the antielectron? Where is the antiproton? A few years after the discovery of the Dirac equation, the antielectron was found in cosmic radiation by Anderson and Blackett. A further quarter of a century was needed before the discovery of the antiproton. This long delay confirmed the belief that the world envisaged by Dirac represented an abstract and peculiar scientific speculation, not intimately connected with our world.

The recent discovery of the existence of only three families of particles was made using a supercollider in which billions upon billions of antielectrons collided with billions upon billions of electrons. If we now know the number of fundamental particles it is due to these gigantic machines. But none of them would exist if the elusive antiparticles predicted by the Dirac equation were not there.

Dirac opened the way towards research for the "useless" particles and we know

\section{Why the proton came to be the antielectron: the true story}

The "useless" particles represented mankind's greatest intellectual achievement and Paul Dirac was the author. $\mathrm{He}$ was also the "author" of an irreverent tale.

As a student, one problem struck me. Pauli, the text-books said, was the physicist

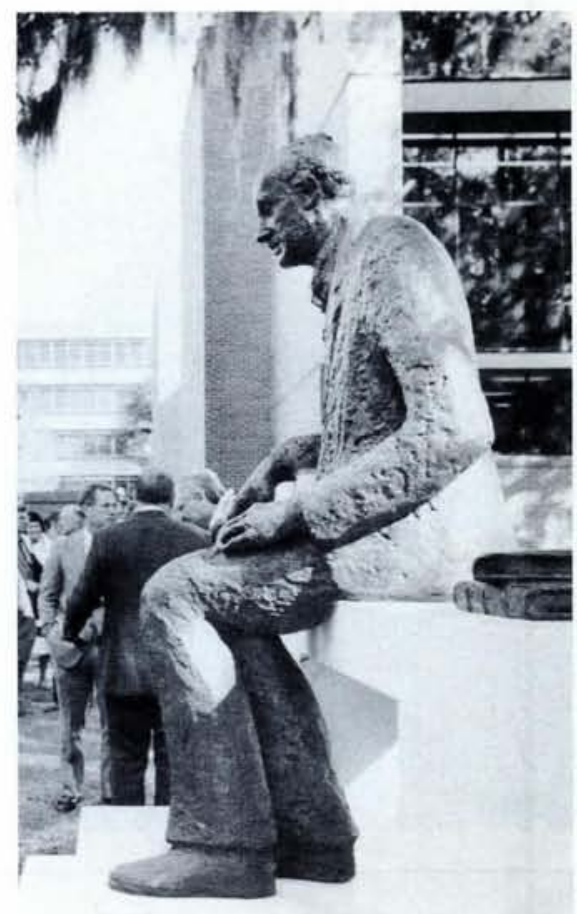

today that there are more useless than useful particles. The world familiar to us is made only with particles belonging to the so-called "first family": the useful one. Nevertheless, it is thanks to the "useless" particles that we are able to trace our existence back to the origin of the universe. In fact, the world opened up by Paul Dirac existed only for a few instants after the big bang. At that time $-15-20$ billion years ago - there was a perfect mixture of particles and antiparticles of all families. Today, after such a long interval of time, the universe has evolved into a world where only particles of the first family exist.

The huge intellectual achievement of Paul Dirac is unprecedented in the history of science: when Galilei studied the way stones run along an inclined plane, the stones were there; when Einstein succeeded in his great synthesis he could see that space-time, mass-energy where there and in front of everybody's eyes; but when Dirac started to think of the existence of the antielectron and of antimatter, nothing of this sort was around. Neither antiparticles nor "useless" particles had ever been observed. Paul Dirac's equation nevertheless tells us that if space-time, mass-energy and matter are there, then antimatter and "useless" particles must also be there.

who pointed out that the antiparticle predicted by the Dirac equation could not be the proton. This is fairly self-evident since the mass of the proton is 2,000 times larger than that of the electron and, in the Dirac equation, the mass of the particle and the antiparticle must be the same. The problem was: how could it be that Dirac himself did not see the discrepancy?

When I had the great privilege of meeting Paul Dirac, I tried to find out how this could have happened. It was for many years a 
hopeless task. Until 1982, when I met Piotr Kapitza who worked in Cambridge with Dirac, his close friend, at the Rutherford Laboratory.

After the discovery of the Dirac equation, at every seminar no matter the topic, someone - often myself, Piotr Kapitza told me - asked: "Dirac, where is the antielectron?". Needless to say, a chorus of laughs closed the seminar.

Paul Dirac eventually had had enough and decided to stop this. He knew that few colleagues were able to follow his arguments, and that even fewer had seriously studied his equation. Wanting to fool all these people, Dirac announced: "The proton has the opposite charge to that of the electron.
So let us take the proton as a good candidate for being the antielectron." And so he started spreading the rumour that he was seriously considering the proton as the antiparticle predicted by his equation. As for its mass, this was a detail that he was studying. Laughing at the end of each seminar stopped as soon as the rumour circulated. No one any longer asked the question, "Dirac, where is the antielectron?".

It was great to hear the story told by Piotr Kapitza, with his humour and ability. Paul Dirac was present and was laughing at Kapitza recollecting the fine hours in Cambridge.

Books carrying Pauli's remarks should perhaps be amended.

\section{IOM Delegates to Council}

The two new delegates of the Individual Ordinary Members to EPS Council, elected following the recent postal ballot, are:

M. Balkanski, Paris (F)

J. Fischer, Prague (CZ)

The delegates remaining in office are:

G. Chiarotti, Rome (I)

P. Choquard, Lausanne $(\mathrm{CH})$

F. Netter, Paris (F)

H. Ryde, Lund (S)

E. Skrzypczak, Warsaw (PL)

D. Stacey, Oxford, (UK)

W.T. Wenckebach, Leiden (NL)

Professor Minko Balkanski is with the Solid State Physics Laboratory, P. and M. Curie University, 4, Place Jussieu, Tour 13, F-75230 Paris Cedex 05. A French citizen born in 1927, he has worked as a Research Fellow at the Fritz Haber Institute in Berlin. A former Director of the Laboratory and a former Chairman of CMD, he remains a member of MIT's Advanced Study Institute.

Dr. Jan Fischer is a senior scientist at the Institute of Physics, Czechoslovak Academy of Sciences, Na Slovance 2, CS-180 40 Prague 8. A Czech citizen born in 1932, he has Doctorates from both the Institute and from Charles University, Prague. He has worked as a visiting scientist at JINR (Dubna), ICTP (Trieste) and CERN; and as a visiting professor at the University of Languedoc, Montpellier. Chief Editor of the Czechoslovak Journal of Physics, he has been an IOM Delegate to Council and is a Member of the HEPP Board.

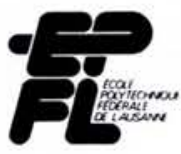

\section{Scientific Collaborators}

The Institute of micro- and opto-electronics of the Swiss Federal Institute of Technology is seeking applications for limited time research positions in the areas of device physics and the properties of heterojunction field effect transistors.

The positions would be suitable for candidates seeking to start a doctoral research program or for recently graduated scientists.

Candidates are asked to apply to:
Prof. M. llegems
Institut de Micro- et Opto- électronique
EPFL
$\mathrm{CH}-1015$ Lausanne
Switzerland

\section{Meeting Announcement}

10-12 Sept. 1991 Lund, Sweden

Nuclear Physics at Storage Rings: Disassembly of Nuclei and Subthreshold Meson Emission

P. Arve, Dept. of Mathematical Physics, Lund Institute of Technology,

POB 118, S-22100 Lund

POB 118, S-22100 Lund
++46 (46) $109090 / 104416$ matfys@ seldc52

\section{Board Changes}

Dr. Thomas Jarlborg has replaced Professor Jean Muller on the Editorial Board of Europhysics News. Swedish and a graduate of Chalmers University, Göteborg, he is an Associate Professor at the Department of Physics of Condensed Matter, University of Geneva where he has worked in the computational group for eight years. Prior to this he spent three years as a postdoc at Northwestern University in the USA. A theoretical solid state physicist, his main research interests involve the application of local density methods to the calculation of electromagnetic properties.

The Executive Committee of EPS has also approved the appointment of Dr. Fredrick James as the next Chairman of the Editorial Board of Europhysics News. An experimental physicist at CERN with a strong interest in data treatment, he is well known to us all as a former Chairman of the Computational Physics Group, and for his activities in connection with the academic freedom of physicists. Dr. James has been on the Board since 1986.

\section{Quantum Electronics Division}

From 1 April 1990, following elections in March, the Board of the Quantum Electronics Division will comprise the members listed below.

\section{Chairman: P.L. Knight, Imperial College, London}

Secretary: J.P. Woerdman, Univ., Leiden

Members:

J.S. Bakos, Central Research Institute, Budapest

R. Bonifacio, Univ., Milan

E. Giacobino, Univ., Paris

E.O. Goebel, Univ., Marburg

D.R. Hall, Heriot-Watt Univ, Edinburgh

J. Mlynek, Univ., Konstanz

O. Poulsen, Univ., Aarhus

F. Pradere, Ecole Poly., Palaiseau

F.K. Rheinhart, EPFL, Lausanne

S. Svanberg, Lund Inst. of Tech.

The Chairman, who took office last October, reports that the Division's main activity involves the organization of the International Quantum Electro- nics Conferences through the International Council of Quantum Electronics.

The 2nd European Quantum Electronics Conference (EOEC 89), which served as the Divisional meeting, was held on 28 August-1 September 1989 in Dresden. The next EQEC will be held in Edinburgh in September 1991.

\section{Environmental Physics Group}

Strong encouragement is emerging for The Institute of Physics plan to form an Environmental Physics Group whose aim is to promote physics within the context of environmental sciences. Support from anyone interested in the Group is welcome as a main objective is liaison with counterparts elsewhere in Europe and further afield. For information, contact Susanna Lithiby, IOP, 47 Belgrave Sq., London SW1X 8QX, Tel.: ++44-71-235 6111 ; Fax: $++44-71-2596002$. 